\title{
REVIEW
}

\section{AN EMERGING TREND IN INFANT FEEDING PRACTICE: A SCOPING REVIEW ON BREASTMILK SHARING}

\author{
Nurul Akma Jamil ${ }^{1}$, Lee Khuan1(D), Ai Theng Cheong ${ }^{2}$, Siti Mariam Muda ${ }^{3}$ \\ ${ }^{1}$ Department of Nursing, Faculty of Medicine and Health Sciences, University Putra Malaysia, Malaysia \\ ${ }^{2}$ Department of Family Medicine, Faculty of Medicine and Health Sciences, University Putra Malaysia, Malaysia \\ ${ }^{3}$ Department of Special Care Nursing, Kulliyyah of Nursing, International Islamic University Malaysia, Malaysia
}

Received July 15, 2020; Accepted December 21, 2020. Copyright: This is an open access article under the CC BY-NC-ND license.

\begin{abstract}
Aim: The aims of this scoping review were to identify the type of available evidence and map rapidly the key concepts underpinning milk sharing research. Design: Scoping review. Methods: Initially, the Scopus, ScienceDirect and EBSCOhost databases were searched. The keywords used were "milk sharing," "expressed breastmilk donation," "peer," "online" and "internet" and a combination of the Boolean operators "AND" and "OR." The snowballing technique was used to identify grey literature. The inclusion criteria were citations in English and Malay languages focusing on milk sharing. The review selection was performed by two independent reviewers. Results: The search identified two theses and 23 journal articles from 2010 to December 2019 that varied in terms of subject areas, aims, and methodologies. The majority of studies included were conducted in Western countries. Key findings identified the emerging concept of shared breastmilk, characteristics of donor and recipient mothers, facilitating factors for milk sharing, an individual's perception versus informed decision and transparency, the perception of "breast is the best" versus a bottle feeding culture, stigma surrounding milk sharing, a lack of involvement of health care providers in decision-making, problems with accessing human milk from the milk bank, and conflicting issues from a religious perspective. Conclusion: Milk sharing is a relatively contemporary infant feeding practice that raises several important issues. However, the existing literature is limited to the exploration of milk sharing practice from a Western perspective. This justifies the need for future research as infant feeding is deeply rooted within religious beliefs and the socio-cultural context.
\end{abstract}

Keywords: breastmilk sharing, expressed breastmilk, infant feeding, scoping review, social network.

\section{Introduction}

Non-maternal nursing originated from wet nursing that existed in many cultures around the world, notably in the ancient Greek and Roman civilizations, as well as during the Industrial Revolution in the 18th and 19th centuries (Campbell, 1989; Fulminante, 2015; Rahbari, 2020; Stevens et al., 2009). Modern-day milk banking, established in the early 20th century in response to the need for infant survival, was inspired by the wet nursing practice (DeMarchis et al., 2017). The modern milk bank and wet nursing are different in terms of regulation and procedure. The milk bank is regulated by a medical institution and abides by a strict standard operating procedure (PATH, 2019). At present, milk banks are only available in Australia, European countries, and the United States. Milk banks are considered highly controversial among the Muslim community due to religious issues (Bawany et al., 2016).

Corresponding author: Lee Khuan, Department of Nursing, Faculty of Medicine and Health Sciences, University Putra Malaysia, 43400 Serdang, Selangor; email: leekhuan@upm.edu.my
With the aid of information technology, non-maternal nursing has been taken one step further. Milk exchanges are now facilitated through websites and social networks. A review of the literature suggested that during the past 10 years, more information has become available on contemporary milk sharing practice (Akre et al., 2011). It corresponds with the establishment of the first internet-based milk sharing organization, Eats on Feets, in 2010. Through its website and Facebook page, women are able to share breastmilk safely and ethically ("Eats on Feets Community Breastmilk Sharing”, 2010).

\section{Aim}

The specific aims of this scoping review were: 1) to conduct a systematic search of published and grey literature; 2) to map the characteristics and range of methodologies used; and 3) to map the literature on the sharing of milk to illuminate the gap in knowledge on this subject. This review was guided by a series of questions with the aim of mapping the literature on milk sharing. The questions were: 1) What are the characteristics and methodologies 
used in the research on milk sharing? 2) What is known from the existing literature about milk sharing?

\section{Methods}

\section{Design}

A scoping review was used due to the scarcity of evidence on the topic under study. A review protocol was developed to maintain the rigour of the review process following the Joanna Brigg's Institute methodology for scoping reviews (Peters et al., 2015).

\section{Eligibility criteria}

The search was limited to research articles published in English and Malay due to lack of resources for translation. Research articles, case studies, and theses were included in this review while conference proceedings, commentaries, books, book series, and book chapters were excluded. A timeframe from 2010 until the present time was used to observe the evolution of research on milk sharing and it was consistent with the emergence of milk sharing in social networks in the 2010s (Akre et al., 2011). Studies that explicitly mention milk donations to institutionalized milk banks or which are related to milk selling and buying activities were also excluded.

\section{Search Strategy}

A multi-method search strategy was employed to ensure that the findings were robust. The search was primarily conducted in August 2018 and repeated in January 2020. Scopus, Science Direct, and EBSCOhost were selected due to their robustness and availability of a wide variety of subject areas. The search strategy was formed upon consultation with librarians. The initial search string consisted of terms such as milk sharing, expressed breastmilk donation, peer, online, and internet. Next, the authors expanded the search process by relying on keywords suggested by the Scopus database: human, breastmilk, breast feeding, milk banks, social media, and social support. Keywords were combined with Boolean operators AND, OR and NOT and the search query was customized to tailor to the specific database requirements. Additionally, the snowballing technique of checking references was used to find relevant citations that were not identified in the initial search. Citations that were found and identified within the search period were included for review.

\section{Study Selection inc. PRISMA flow diagram}

Figure 1 shows the flow diagram for the literature search in this review. Initially, an electronic database search captured a total of 87 records, while 20 other records were obtained through the snowballing technique from the reference lists of the latest papers published. All citations found from the search were transferred to Mendeley, web-based reference manager software. A deduplication of records between databases yielded 74 records. Seventy-four articles with appropriate titles and abstracts were screened independently by $\mathrm{JN}$ and LK. At that point, 33 records were removed due to irrelevance. After attempts were made to contact the source author, one complete citation could not be obtained. The remaining 40 records were screened for eligibility based on the inclusion and exclusion criteria. At that stage, 15 records were excluded; two case studies that implemented a blended concept of breastmilk donation and wet nursing practice but used the term milk sharing, three studies on milk purchasing via public social networks, nine commentaries, position statements and editorial notes, and one experimental study. A total of 23 journal articles and two theses were found to be eligible for inclusion in the review.

\section{Evaluation of quality of articles}

All 23 journal articles included in this review were methodologically varied; a universal appraisal tool developed by Hawker et al. (2002) was used to assess the quality of the papers in general. This appraisal tool comprises of nine components that allow evaluation of the clarity of the information in the abstract and title section, introduction and aims, method and data, sampling, data analysis, ethics and bias, results, transferability or generalizability of the findings, and its implications to the policy and practice. The evaluation of the quality of articles was done independently by NA and discussed by all authors. The majority of the studies were considered high quality as they scored more than 30 points. The details of the quality scores of included articles against Hawker's appraisal tool are presented in Table 1.

\section{Data extraction}

The findings are presented according to the process where primary attention is given to a basic analysis of the extent, nature, and distribution of included studies. A systematic approach was used to describe, classify, and conceptualize the information obtained from the included citations. The authors created an analytical structure representing the aims of this analysis, consisting of the first author's name, publication year, location of the research, nature of the study, sample size, sampling technique, methods and main findings. Then the mapping process was carried out by JN and LK where any differences would be discussed by all authors to obtain clarity. 


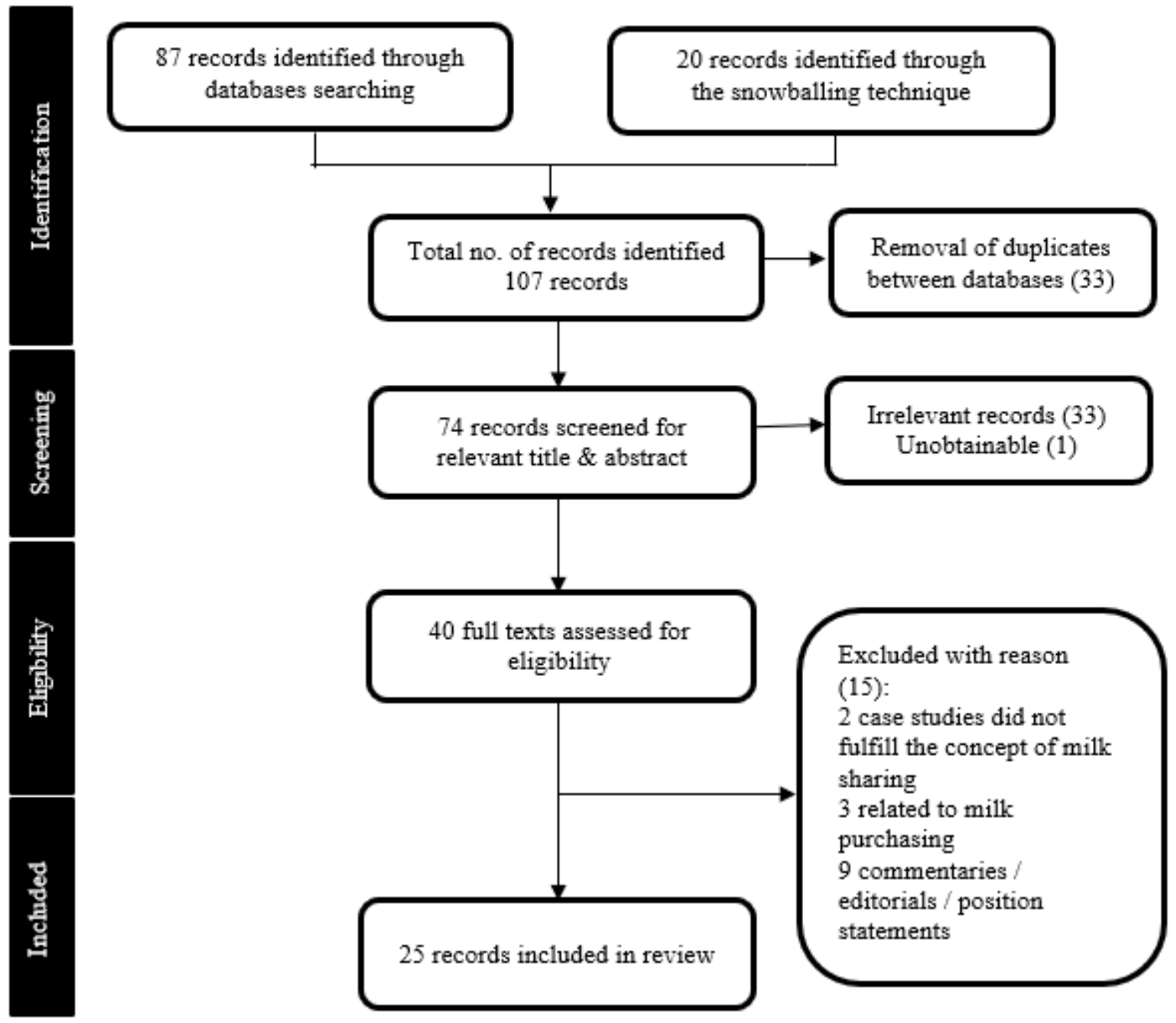

Figure 1 Flow diagram of study selection, following the PRISMA guideline

\section{Results}

\section{General characteristics of included citations}

The majority of citations used in this review were journal articles. In terms of subject areas, a majority of the included studies were on health science, with only five on social science and one on human science. This review was based on 25 studies in three countries / regions: 17 studies were conducted in the United States and North America, seven in Australia and one in Turkey. In terms of the years of publication, one was published in 2012, two were published in 2013, six were published in 2014, two were published in 2015, five were published in 2016, two were published in 2017, six were published in 2018, and one was published in 2019.

\section{Methodological characteristics of included citations}

In terms of methodology that underpinned the existing studies, eight of them were quantitative, 13 were qualitative, and four were mixed-method studies. From the 13 qualitative studies, two were discourse analyses while the rest applied various research paradigms as follows: eight descriptive qualitative, two ethnographic, and one grounded theory study. The qualitative studies also employed triangulation via use of different methods (Smith, 2017), different types of informants (Gribble, 2013; 2014a; 2014b; 2018; McCloskey \& Karandikar, 2018; O'Sullivan et al., 2016; Smith, 2017), use of purposive sampling (Carter et al., 2015; Smith, 2017; Thorley, 2012), and adoption of well-recognized qualitative data analysis (Carter \& Reyes-Foster, 2016; Gribble, 2013; 2014a; 2014b; 2018; MacDonald et al., 2016; Papanicolaou, 2013; Perrin et al., 2016; Smith, 2017; Thorley, 2012).

Similarly, mixed-method studies included in this review also incorporated strong quantitative and qualitative components such as the application of overlapping methods while collecting data (ReyesFoster \& Carter, 2018), adoption of well-established 
qualitative data analysis (Perrin et al., 2014; ReyesFoster \& Carter, 2018), and large sample sizes from multiple settings (Palmquist \& Doehler, 2016; Perrin et al., 2014; Reyes-Foster \& Carter, 2018).

In the same way, the strengths of quantitative studies lie in the use of validated instruments for data collection (Cassar-Uhl \& Liberatos, 2018; Keim et al., 2014; Onat \& Karakoç, 2019), recruitment of large samples (Cassar-Uhl \& Liberatos, 2018; Gribble, 2014c; Onat \& Karakoç, 2019, Palmquist \& Doehler, 2014; Reyes-Foster et al., 2015; 2017; Schafer et al., 2018), and involvement of participants from multiple settings (Keim et al., 2014). Details of the methodological characteristics of citations included in this scoping review are presented in Table 1.

\section{Key findings across the literature}

The key findings of this review are summarized in Figure 2.

\section{The concept of milk sharing}

The authors found that the terms informal milk sharing (Onat \& Karakoç, 2019; O’Sullivan et al., 2016), peerto-peer milk sharing (Carter et al., 2018; Gribble, 2014a, 2014b; McCloskey \& Karandikar, 2018; Perrin et al., 2016; Reyes-Foster et al., 2017), milk sharing via the internet (Keim et al., 2014) and internet-based peer-to-peer milk sharing (Gribble, 2013, 2018) were used across the literature. Regardless of the term used, it refers to the milk exchange activity that is facilitated by social networks. These social networks serve as a platform where mothers can request and donate expressed breastmilk and the milk is freely provided and collected for feeding a recipient child.

\section{The characteristics of donor and recipient mothers}

Milk sharing is predominantly practiced by young mothers who are within the reproductive age group, possess a high level of education, are employed, gave birth to a single baby, and mothers of premature babies (Carter et al., 2018; Cassar-Uhl \& Liberatos, 2018; Gribble, 2013, 2014a, 2014b; McCloskey \& Karandikar, 2018; Papanicolaou, 2013; Perrin et al., 2016; Reyes-Foster \& Carter, 2018; Reyes-Foster et al., 2017; Schafer et al., 2018; Smith, 2017; Thorley, 2012). However, there are slight differences in terms of the age range of participants across the literature. It varies from 18 to 58 years (Reyes-Foster et al., 2017; Schafer et al., 2018) to 21 to 45 years (Gribble, 2013, 2014a, 2014c; McCloskey \& Karandikar, 2018). A discrepancy in maternal parity was also seen in the study by Reyes-Foster et al. (2017) where $61.5 \%$ of the participants were multigravida. Additionally, milk sharing has become a choice in infant feeding practice for adoptive parenting (Palmquist \& Doehler, 2016).

\section{The facilitating factors of milk sharing}

The facilitating factors involved in milk sharing are categorized into three aspects: the donor mother, recipient mother, and recipient infant. Perception of the significant value of breastmilk, altruism, surplus breastmilk, staying apart from the biological child or the child's death are the codes that make up this category. From the donor's perspective, the ability to produce extra breastmilk and the perception of the significant value of breastmilk motivate them to participate in milk sharing, and a sense of satisfaction is derived from helping infants in need (Gribble, 2013; Perrin et al., 2016). This perception causes them to be selective in choosing to whom their milk should be given and they prefer milk sharing via social networks over donating it to the institutionalized milk bank. In addition, excellent communication between donor mothers and recipient mothers would establish trust and enable a relationship to be developed between them; this is particularly important among Muslims. Mothers' concerns on this issue were highlighted in studies by Gribble (2013) and Thorley (2012). Therefore, being in touch following milk sharing is vital to avoid problems related to milk kinship.

The concerns of recipient mothers include the inability to breastfeed or produce breastmilk, breastfeeding difficulties, experience of using infant formula, feeling guilty for not providing breastmilk, perception of the value of breastmilk, religious order, and the nature of being a mother (Carter et al., 2015; Gribble, 2013; Keim et al., 2014; McCloskey \& Karandikar, 2018; O'Sullivan et al., 2016; Perrin et al., 2014; Schafer et al., 2018; Smith, 2017; Thorley, 2012). Carter et al. (2015) and MacDonald et al. (2016) also state that milk sharing has become a solution for lesbian, gay, bisexual, transgender, queer, two-spirits, and other persons who engage in parenting. Infants' health conditions such as failure to thrive, formula intolerance, food allergy, and tongue-tie have been found to influence mothers' decision to choose milk sharing (Gribble, 2014b; Palmquist \& Doehler, 2014; Thorley, 2012). In contrast, Carter et al. (2015) found that infants' health problems are not the main factor, as feeding an infant using non-maternal breastmilk is a parental choice rather than a medical necessity. The findings are supported by a recent study claiming that feeding of an infant by adoptive parents using the infant's non-biological mother's milk is influenced by the sense of "being a mother" (Carter et al., 2018).

An individual's perception versus informed decisions and transparency

Safety issues pose the main challenges in milk sharing practice. Therefore, both parties, that is the donor and the recipient, adopt several strategies to mitigate the 
Table 1 Methodological characteristics of included studies (Part 1)

\begin{tabular}{|c|c|c|c|c|c|c|c|c|}
\hline 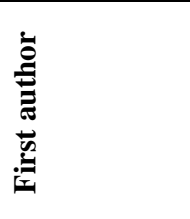 & $\stackrel{\bar{\Xi}}{\grave{J}}$ & $\stackrel{\Xi}{E}$ & 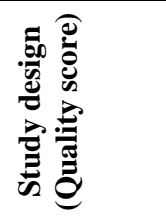 & 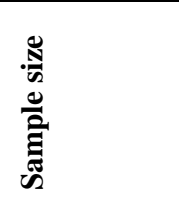 & 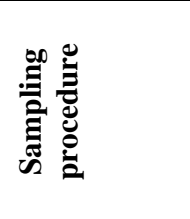 & 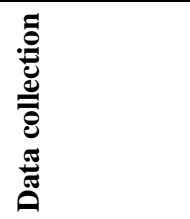 & 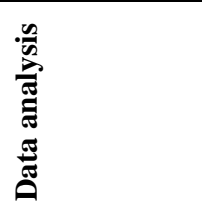 & $\stackrel{\mathscr{E}}{:}$ \\
\hline Onat & 2019 & Turkey & $\begin{array}{l}\text { quantitative } \\
\text { (26) }\end{array}$ & $\begin{array}{l}435(371 \\
\text { non-milk } \\
\text { sharers, } 48 \\
\text { milk donors, } \\
16 \text { milk } \\
\text { recipients) }\end{array}$ & $\begin{array}{l}\text { convenience } \\
\text { sampling of } \\
\text { mothers in a } \\
\text { social media } \\
\text { group }\end{array}$ & online survey & $\begin{array}{l}\text { descriptive } \\
\text { statistic }\end{array}$ & $\begin{array}{l}\text { religious concern } \\
\text { is the barrier to } \\
\text { share milk among } \\
\text { Muslims; } \\
\text { strategies taken } \\
\text { to adhere to } \\
\text { religious rules }\end{array}$ \\
\hline Reyes-Foster & 2018 & US & $\begin{array}{l}\text { mixed } \\
\text { method } \\
(32)\end{array}$ & 390 & $\begin{array}{l}\text { convenience } \\
\text { sampling for } \\
\text { the } \\
\text { quantitative } \\
\text { part; } \\
\text { purposive } \\
\text { sampling for } \\
\text { the } \\
\text { qualitative } \\
\text { part }\end{array}$ & $\begin{array}{l}\text { observation, } \\
\text { semi- } \\
\text { structured } \\
\text { interview, } \\
\text { online survey }\end{array}$ & $\begin{array}{l}\text { quantitative: } \\
\text { descriptive } \\
\text { statistic } \\
\text { qualitative: } \\
\text { grounded } \\
\text { theory analysis }\end{array}$ & $\begin{array}{l}\text { distinguished } \\
\text { features between } \\
\text { milk exchange \& } \\
\text { milk selling; } \\
\text { importance of } \\
\text { safe milk } \\
\text { handling practice }\end{array}$ \\
\hline Cassar-Uhl & 2018 & US & $\begin{array}{l}\text { quantitative } \\
\text { (32) }\end{array}$ & 475 & $\begin{array}{l}\text { voluntary } \\
\text { sampling of } \\
\text { mothers in } \\
\text { social media } \\
\text { self- } \\
\text { identified as } \\
\text { having low } \\
\text { milk supply }\end{array}$ & online survey & $\begin{array}{l}\text { descriptive } \& \\
\text { chi square test }\end{array}$ & $\begin{array}{l}\text { milk sharing is } \\
\text { high among } \\
\text { mothers with low } \\
\text { milk supply }\end{array}$ \\
\hline Carter & 2018 & US & $\begin{array}{l}\text { mixed } \\
\text { method } \\
(34)\end{array}$ & 392 & $\begin{array}{l}\text { voluntary } \\
\text { sampling of } \\
\text { mothers in } \\
\text { online } \\
\text { breastfeeding } \\
\text { and milk } \\
\text { sharing } \\
\text { groups }\end{array}$ & online survey & $\begin{array}{l}\text { quantitative: } \\
\text { descriptive } \\
\text { statistic } \\
\text { qualitative: } \\
\text { social } \\
\text { constructionist }\end{array}$ & $\begin{array}{l}\text { mothers' } \\
\text { perception of the } \\
\text { value of } \\
\text { breastmilk } \\
\text { influenced them } \\
\text { to choose milk } \\
\text { sharing over } \\
\text { formula feeding; } \\
\text { feeding infants } \\
\text { using non- } \\
\text { biological } \\
\text { mothers' milk is } \\
\text { influenced by the } \\
\text { sense of "being a } \\
\text { mother" }\end{array}$ \\
\hline Schafer & 2018 & US & $\begin{array}{l}\text { quantitative } \\
\text { (34) }\end{array}$ & 205 & $\begin{array}{l}\text { voluntary } \\
\text { sampling of } \\
\text { milk } \\
\text { recipients }\end{array}$ & online survey & $\begin{array}{l}\text { descriptive \& } \\
\text { multivariate } \\
\text { analyses }\end{array}$ & $\begin{array}{l}\text { breastfeeding } \\
\text { difficulty } \\
\text { associated with } \\
\text { negative } \\
\text { emotions; } \\
\text { milk sharing } \\
\text { associated with } \\
\text { social stigma }\end{array}$ \\
\hline
\end{tabular}


Table 1 Methodological characteristics of included studies (Part 2)

\begin{tabular}{|c|c|c|c|c|c|c|c|c|}
\hline 离 & $\stackrel{\varpi}{\nexists}$ & $\stackrel{\Xi}{\Xi}$ & 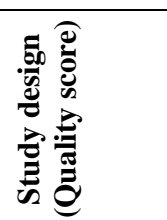 & 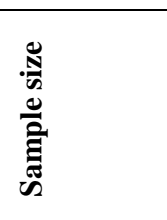 & 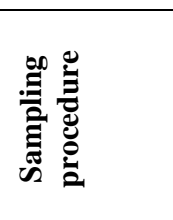 & 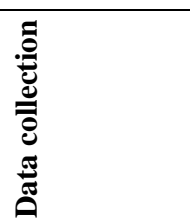 & 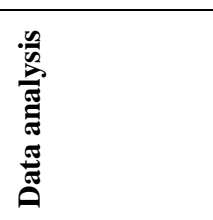 & $\stackrel{\mathscr{0}}{\mathscr{E}}$ \\
\hline McCloskey & 2018 & US & $\begin{array}{l}\text { qualitative } \\
\text { (basic) } \\
(36)\end{array}$ & 20 & $\begin{array}{l}\text { convenience } \\
\text { sampling of } \\
\text { women who } \\
\text { had } \\
\text { experiences } \\
\text { with milk } \\
\text { sharing }\end{array}$ & $\begin{array}{l}\text { semi- } \\
\text { structured } \\
\text { online } \\
\text { interview }\end{array}$ & $\begin{array}{l}\text { grounded } \\
\text { theory analysis }\end{array}$ & $\begin{array}{l}\text { challenges that } \\
\text { influence } \\
\text { mothers to be } \\
\text { involved in milk } \\
\text { sharing are due } \\
\text { to difficulties in } \\
\text { accessing } \\
\text { breastmilk from } \\
\text { the milk bank; } \\
\text { lack of societal } \\
\text { acceptance of } \\
\text { milk sharing } \\
\text { leads to stigma; } \\
\text { facilitators for } \\
\text { milk sharing: } \\
\text { informed } \\
\text { decision-making } \\
\text { \& transparency } \\
\text { from both parties } \\
\text { \& support } \\
\text { received from } \\
\text { health care } \\
\text { providers }\end{array}$ \\
\hline Reyes-Foster & 2017 & US & $\begin{array}{l}\text { quantitative } \\
\text { (34) }\end{array}$ & 321 & $\begin{array}{l}\text { convenience } \\
\text { sampling of } \\
\text { individuals } \\
\text { in the milk } \\
\text { sharing } \\
\text { community }\end{array}$ & online survey & $\begin{array}{l}\text { descriptive, } \\
\text { univariate \& } \\
\text { multivariate } \\
\text { analyses }\end{array}$ & $\begin{array}{l}\text { milk sharers } \\
\text { have good } \\
\text { handling practice } \\
\text { of expressed } \\
\text { human milk }\end{array}$ \\
\hline Carter & 2016 & US & $\begin{array}{l}\text { qualitative } \\
\text { (discourse } \\
\text { analysis) } \\
(32)\end{array}$ & $\begin{array}{l}34 \\
\text { newspapers }\end{array}$ & $\begin{array}{l}\text { purposive } \\
\text { sampling }\end{array}$ & - & $\begin{array}{l}\text { interpretive } \\
\text { analysis }\end{array}$ & $\begin{array}{l}\text { milk banks are } \\
\text { portrayed as } \\
\text { receiving } \\
\text { institutional } \\
\text { support from the } \\
\text { government and } \\
\text { medical } \\
\text { institution; } \\
\text { peer milk } \\
\text { sharing is } \\
\text { portrayed as } \\
\text { receiving } \\
\text { warning from } \\
\text { various } \\
\text { institutions due } \\
\text { to the risks }\end{array}$ \\
\hline Palmquist & 2016 & US & $\begin{array}{l}\text { mixed } \\
\text { method } \\
(36)\end{array}$ & $\begin{array}{l}661 \text { donors, } \\
206 \\
\text { recipients }\end{array}$ & $\begin{array}{l}\text { voluntary } \\
\text { sampling }\end{array}$ & online survey & $\begin{array}{l}\text { descriptive \& } \\
\text { univariate } \\
\text { analyses }\end{array}$ & $\begin{array}{l}\text { participants } \\
\text { perception on } \\
\text { the risk of milk } \\
\text { sharing is } \\
\text { reflected by } \\
\text { strategies taken } \\
\text { to minimize the } \\
\text { risk }\end{array}$ \\
\hline
\end{tabular}


Table 1 Methodological characteristics of included studies (Part 3)

\begin{tabular}{|c|c|c|c|c|c|c|c|c|}
\hline 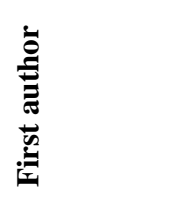 & 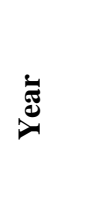 & $\stackrel{\Xi}{\Xi}$ & 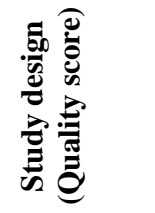 & 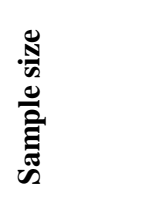 & 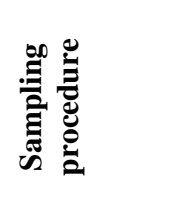 & 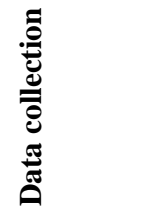 & 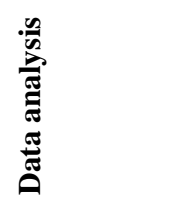 & 总 \\
\hline O'Sullivan & 2016 & US & $\begin{array}{l}\text { qualitative } \\
\text { (basic) } \\
(36)\end{array}$ & 41 & $\begin{array}{l}\text { purposive } \\
\text { sampling of } \\
\text { mothers } \\
\text { who had } \\
\text { experiences } \\
\text { with milk } \\
\text { sharing }\end{array}$ & $\begin{array}{l}\text { semi- } \\
\text { structured, } \\
\text { face-to-face } \\
\text { interview }\end{array}$ & $\begin{array}{l}\text { thematic } \\
\text { analysis }\end{array}$ & $\begin{array}{l}\text { awareness of } \\
\text { human milk } \\
\text { sharing; } \\
\text { consideration of } \\
\text { human milk } \\
\text { sharing; } \\
\text { concerns about } \\
\text { human milk } \\
\text { sharing; } \\
\text { motivations for } \\
\text { human milk } \\
\text { sharing; } \\
\text { routes of human } \\
\text { milk sharing }\end{array}$ \\
\hline Perrin & 2016 & US & $\begin{array}{l}\text { qualitative } \\
\text { (grounded } \\
\text { theory) } \\
(36)\end{array}$ & 27 & $\begin{array}{l}\text { stratified } \\
\text { purposive } \\
\text { sampling }\end{array}$ & $\begin{array}{l}\text { semi- } \\
\text { structured } \\
\text { telephone } \\
\text { interview }\end{array}$ & $\begin{array}{l}\text { constant } \\
\text { comparison } \\
\text { analysis }\end{array}$ & $\begin{array}{l}\text { perception of the } \\
\text { goodness of } \\
\text { breastmilk, } \\
\text { sources of } \\
\text { information } \\
\text { regarding milk } \\
\text { exchange, } \\
\text { concerns \& } \\
\text { knowledge about } \\
\text { milk sharing \& } \\
\text { the sense of } \\
\text { helping the others }\end{array}$ \\
\hline MacDonald & 2016 & US & $\begin{array}{l}\text { qualitative } \\
\text { (basic) } \\
(36)\end{array}$ & 22 & $\begin{array}{l}\text { convenience } \\
\text { sampling of } \\
\text { individuals } \\
\text { self- } \\
\text { identified as } \\
\text { transmasculi } \\
\text { ne }\end{array}$ & $\begin{array}{l}\text { online } \\
\text { interview }\end{array}$ & $\begin{array}{l}\text { interpretive } \\
\text { analysis }\end{array}$ & $\begin{array}{l}\text { participants' } \\
\text { experiences of } \\
\text { gender dysphoria, } \\
\text { chest } \\
\text { masculinization } \\
\text { surgery before } \\
\text { pregnancy or } \\
\text { after weaning, } \\
\text { accessing } \\
\text { lactation care as a } \\
\text { transmasculine } \\
\text { person }\end{array}$ \\
\hline Carter & 2015 & US & $\begin{array}{l}\text { qualitative } \\
\text { (discourse } \\
\text { analysis) } \\
(34)\end{array}$ & $\begin{array}{l}30 \\
\text { newspaper } \\
\text { articles }\end{array}$ & $\begin{array}{l}\text { purposive } \\
\text { sampling }\end{array}$ & - & $\begin{array}{l}\text { feminist } \\
\text { critical } \\
\text { discourse }\end{array}$ & $\begin{array}{l}\text { breastmilk from } \\
\text { the milk bank is } \\
\text { viewed as } \\
\text { lifesaving, milk } \\
\text { donation is } \\
\text { altruistic, } \\
\text { obtaining milk is } \\
\text { a responsible } \\
\text { action; } \\
\text { breastmilk } \\
\text { obtained via milk } \\
\text { sharing has risks, } \\
\text { mothers involved } \\
\text { are not fully } \\
\text { informed of the } \\
\text { risks \& it is an } \\
\text { irresponsible } \\
\text { decision }\end{array}$ \\
\hline
\end{tabular}


Table 1 Methodological characteristics of included studies (Part 4)

\begin{tabular}{|c|c|c|c|c|c|c|c|c|}
\hline 童 & 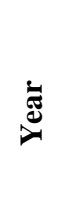 & 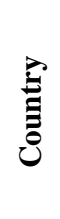 & 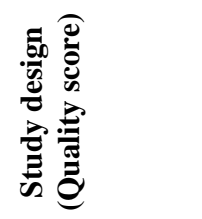 & 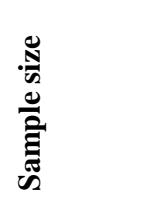 & 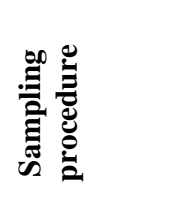 & 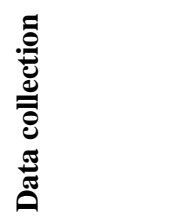 & 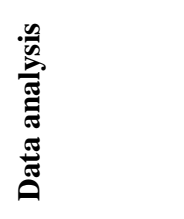 & $\stackrel{\mathscr{E}}{:}$ \\
\hline Reyes-Foster & 2015 & US & $\begin{array}{l}\text { quantitative } \\
\text { (34) }\end{array}$ & 392 & $\begin{array}{l}\text { convenience } \\
\text { sampling }\end{array}$ & online survey & $\begin{array}{l}\text { descriptive \& } \\
\text { univariate } \\
\text { analyses }\end{array}$ & $\begin{array}{l}\text { milk sharing is } \\
\text { complex, donor } \\
\& \text { recipient } \\
\text { mothers are } \\
\text { overlapping; } \\
\text { cross nursing \& } \\
\text { milk exchange } \\
\text { occurs } \\
\text { concurrently }\end{array}$ \\
\hline Keim & 2014 & US & $\begin{array}{l}\text { quantitative } \\
\text { (32) }\end{array}$ & $\begin{array}{l}254 \\
\text { postings } \\
\text { on milk } \\
\text { sharing } \\
\text { websites }\end{array}$ & $\begin{array}{l}\text { purposive } \\
\text { sampling }\end{array}$ & $\begin{array}{l}\text { milk sharing } \\
\text { postings }\end{array}$ & $\begin{array}{l}\text { descriptive, } \\
\text { univariate, \& } \\
\text { bivariate } \\
\text { analyses }\end{array}$ & $\begin{array}{l}\text { contents of the } \\
\text { postings include } \\
\text { the purpose of } \\
\text { donating / } \\
\text { seeking milk, } \\
\text { health behaviour, } \\
\text { milk handling } \\
\text { practice \& } \\
\text { strategy taken to } \\
\text { minimize the } \\
\text { risk; } \\
\text { the readability of } \\
\text { the content was } \\
\text { measured using } \\
\text { Flesch-Kincaid } \\
\text { Grade Level }\end{array}$ \\
\hline Perrin & 2014 & US & $\begin{array}{l}\text { mixed method } \\
\text { (36) }\end{array}$ & $\begin{array}{l}\text { 3-month } \\
\text { facebook } \\
\text { postings }\end{array}$ & $\begin{array}{l}\text { universal } \\
\text { sampling }\end{array}$ & $\begin{array}{l}\text { milk sharing } \\
\text { posting }\end{array}$ & $\begin{array}{l}\text { qualitative } \\
\text { content } \\
\text { analysis }\end{array}$ & $\begin{array}{l}\text { the process of } \\
\text { milk exchange in } \\
\text { the social } \\
\text { network }\end{array}$ \\
\hline Palmquist & 2014 & US & $\begin{array}{l}\text { quantitative } \\
\text { (32) }\end{array}$ & $\begin{array}{l}661 \text { milk } \\
\text { donors, } \\
206 \text { milk } \\
\text { recipients }\end{array}$ & $\begin{array}{l}\text { voluntary } \\
\text { sampling }\end{array}$ & online survey & $\begin{array}{l}\text { descriptive \& } \\
\text { parametric } \\
\text { tests }\end{array}$ & $\begin{array}{l}\text { both donors and } \\
\text { recipients } \\
\text { reported higher } \\
\text { than the national } \\
\text { average for } \\
\text { household } \\
\text { income, maternal } \\
\text { educational } \\
\text { attainment, } \\
\text { breastfeeding } \\
\text { exclusivity 0-6 } \\
\text { months, and } \\
\text { breastfeeding } \\
\text { duration }\end{array}$ \\
\hline Papanicolaou & 2013 & US & $\begin{array}{l}\text { qualitative } \\
\text { (basic) } \\
\text { (not } \\
\text { applicable) }\end{array}$ & 13 & $\begin{array}{l}\text { purposive } \\
\text { sampling of } \\
\text { women in the } \\
\text { milk sharing } \\
\text { community }\end{array}$ & $\begin{array}{l}\text { semi- } \\
\text { structured } \\
\text { online \& } \\
\text { offline } \\
\text { interviews }\end{array}$ & $\begin{array}{l}\text { qualitative } \\
\text { content } \\
\text { analysis }\end{array}$ & $\begin{array}{l}\text { commitment to } \\
\text { provide } \\
\text { breastmilk, } \\
\text { virtual nature of } \\
\text { relationship \& } \\
\text { making the } \\
\text { private public }\end{array}$ \\
\hline
\end{tabular}


Table 1 Methodological characteristics of included studies (Part 5)

\begin{tabular}{|c|c|c|c|c|c|c|c|c|}
\hline 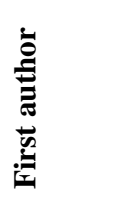 & $\underset{\nabla}{\bar{j}}$ & $\stackrel{己}{\Xi}$ & 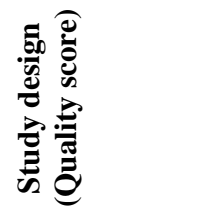 & 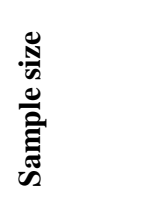 & 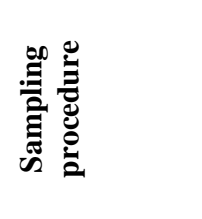 & 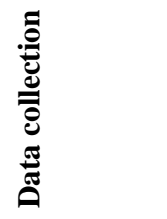 & 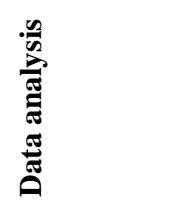 & $\stackrel{600}{:}$ \\
\hline Gribble & 2018 & Australia & $\begin{array}{l}\text { qualitative } \\
\text { (basic) } \\
(35)\end{array}$ & $\begin{array}{l}97 \text { milk } \\
\text { donors, } 41 \\
\text { milk } \\
\text { recipients }\end{array}$ & $\begin{array}{l}\text { convenience } \\
\text { sampling }\end{array}$ & $\begin{array}{l}\text { online } \\
\text { survey }\end{array}$ & $\begin{array}{l}\text { conventional } \\
\text { content } \\
\text { analysis }\end{array}$ & $\begin{array}{l}\text { developing } \\
\text { personal } \\
\text { relationship } \\
\text { between milk } \\
\text { sharers is } \\
\text { important; } \\
\text { milk kinship } \\
\text { established } \\
\text { following milk } \\
\text { sharing }\end{array}$ \\
\hline Smith & 2017 & Australia & $\begin{array}{l}\text { qualitative } \\
\text { (ethnographic) } \\
\text { (not } \\
\text { applicable) }\end{array}$ & 13 & $\begin{array}{l}\text { purposive } \\
\text { sampling of } \\
\text { women who } \\
\text { experience } \\
\text { milk } \\
\text { insufficiency }\end{array}$ & $\begin{array}{l}\text { in-depth } \\
\text { interview, } \\
\text { observation }\end{array}$ & $\begin{array}{l}\text { grounded } \\
\text { theory } \\
\text { analysis }\end{array}$ & $\begin{array}{l}\text { milk sharing is } \\
\text { an alternative } \\
\text { for women that } \\
\text { experience milk } \\
\text { insufficiency }\end{array}$ \\
\hline Gribble & $2014 a$ & Australia & $\begin{array}{l}\text { qualitative } \\
\text { (basic) } \\
(35)\end{array}$ & $\begin{array}{l}97 \text { milk } \\
\text { donors }\end{array}$ & $\begin{array}{l}\text { convenience } \\
\text { sampling }\end{array}$ & $\begin{array}{l}\text { online } \\
\text { survey }\end{array}$ & $\begin{array}{l}\text { conventional } \\
\text { content } \\
\text { analysis }\end{array}$ & $\begin{array}{l}\text { milk sharing is } \\
\text { motivated by } \\
\text { helping the } \\
\text { others }\end{array}$ \\
\hline Gribble & $2014 b$ & Australia & $\begin{array}{l}\text { qualitative } \\
\text { (basic) } \\
(34)\end{array}$ & $\begin{array}{l}41 \text { milk } \\
\text { recipients }\end{array}$ & $\begin{array}{l}\text { convenience } \\
\text { sampling of } \\
\text { milk } \\
\text { recipients on a } \\
\text { facebook page }\end{array}$ & $\begin{array}{l}\text { online } \\
\text { survey }\end{array}$ & $\begin{array}{l}\text { conventional } \\
\text { content } \\
\text { analysis }\end{array}$ & $\begin{array}{l}\text { milk sharing is } \\
\text { an alternative } \\
\text { when mothers } \\
\text { face } \\
\text { breastfeeding } \\
\text { difficulties }\end{array}$ \\
\hline Gribble & $2014 c$ & Australia & $\begin{array}{l}\text { quantitative } \\
\text { (32) }\end{array}$ & $\begin{array}{l}97 \text { milk } \\
\text { donors, } 41 \\
\text { milk } \\
\text { recipients }\end{array}$ & $\begin{array}{l}\text { convenience } \\
\text { sampling of } \\
\text { milk sharers } \\
\text { in the milk } \\
\text { sharing } \\
\text { community }\end{array}$ & $\begin{array}{l}\text { online } \\
\text { survey }\end{array}$ & $\begin{array}{l}\text { conventional } \\
\text { content } \\
\text { analysis }\end{array}$ & $\begin{array}{l}\text { milk sharing } \\
\text { provides an } \\
\text { alternative } \\
\text { solution for } \\
\text { mothers who } \\
\text { are unable to } \\
\text { breastfeed; } \\
\text { strategies to } \\
\text { minimize health } \\
\text { risks; } \\
\text { for Muslim } \\
\text { mothers, } \\
\text { additional } \\
\text { concerns related } \\
\text { to religious } \\
\text { rules }\end{array}$ \\
\hline Gribble & 2013 & Australia & $\begin{array}{l}\text { qualitative } \\
\text { (basic) } \\
(36)\end{array}$ & $\begin{array}{l}98 \text { milk } \\
\text { donors, } 41 \\
\text { milk } \\
\text { recipients }\end{array}$ & $\begin{array}{l}\text { convenience } \\
\text { sampling }\end{array}$ & $\begin{array}{l}\text { online } \\
\text { survey }\end{array}$ & $\begin{array}{l}\text { conventional } \\
\text { content } \\
\text { analysis }\end{array}$ & $\begin{array}{l}\text { milk sharing is } \\
\text { an alternative } \\
\text { when the milk } \\
\text { bank is not } \\
\text { available; } \\
\text { mothers donate } \\
\text { to a peer using } \\
\text { internet-based } \\
\text { milk sharing } \\
\text { networks due to } \\
\text { doubts about the } \\
\text { process in the } \\
\text { milk bank }\end{array}$ \\
\hline Thorley & 2012 & Australia & $\begin{array}{l}\text { qualitative } \\
\text { (ethnographic) } \\
\text { (35) }\end{array}$ & 22 & $\begin{array}{l}\text { purposive } \\
\text { sampling }\end{array}$ & $\begin{array}{l}\text { online } \\
\text { interview }\end{array}$ & $\begin{array}{l}\text { thematic } \\
\text { analysis }\end{array}$ & $\begin{array}{l}\text { cultural issues } \\
\text { related to milk } \\
\text { and consent }\end{array}$ \\
\hline
\end{tabular}


risks. This is manifested in milk sharing advertisements on the internet that provide information concerning the donors' health status, lifestyle, and their hygiene and handling practice (Gribble, 2014c; Keim et al., 2014; Onat \& Karakoç, 2019; Palmquist \& Doehler, 2016; Reyes-Foster \& Carter, 2018; Reyes-Foster et al., 2017). For Muslim milk sharers, additional aspects are reported to uphold the Islamic religious principle. They deal with their concerns regarding the implications of milk kinship by finding infants of the same gender, limiting the number of donors or recipients, and getting to know each other (Onat \& Karakoç, 2019; Thorley, 2012).

The perception of "breast is the best" versus a bottlefeeding culture

It was learnt that the mothers' decision to use nonbiological mother's milk is driven by their awareness of its nutritional benefits (Carter \& Reyes-Foster, 2016) and it is intensified by the mother's desire to breastmilk, as breastfeeding has been perceived as a symbol of maternal love (Carter et al., 2018; Gribble, 2018; MacDonald et al., 2016; Smith, 2017).

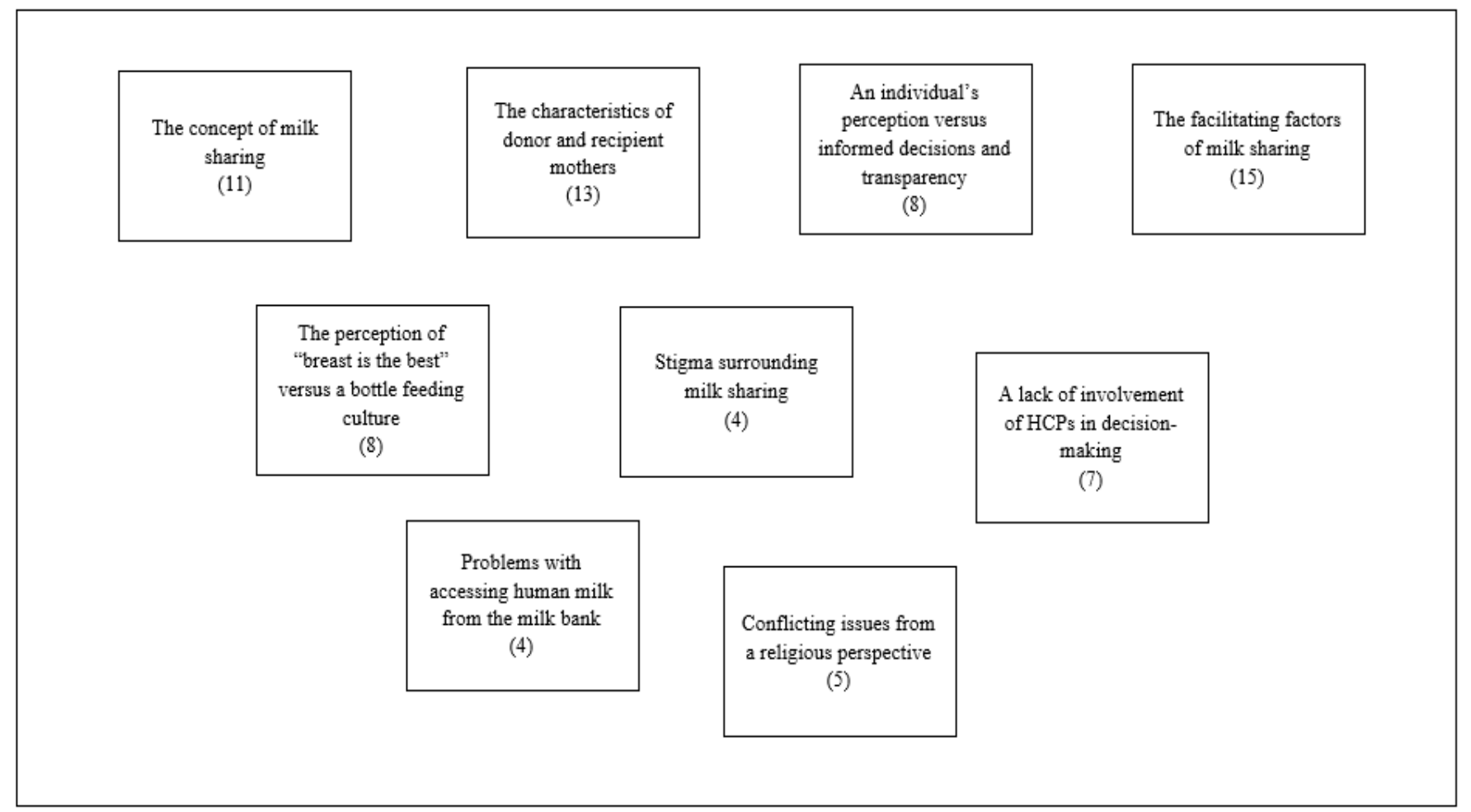

Figure 2 Key findings of the review measured by numbers of studies

This perception, however, might present complications as mothers may experience challenges to sustain the breastfeeding practice. Therefore, feeding their infants using non-maternal breastmilk seems to be an alternative for these unfortunate mothers (McCloskey \& Karandikar, 2018; Schafer et al., 2018; Thorley, 2012).

\section{Stigma surrounding milk sharing}

Stigma that exists around milk sharing is due to a lack of awareness and acceptance of milk sharing. This stigma may negatively influence the emotional responses of recipient mothers (Schafer et al., 2018). In contrast, the existing literature also indicates that providing milk is associated with positive attitudes when compared to receiving it (Onat \& Karakoç, 2019; McCloskey \& Karandikar, 2018; O’Sullivan et al., 2016).
A lack of involvement of health care providers (HCPs) in decision-making

An individual's decision to participate in milk sharing without medical consideration would result in adverse effects to the donor and recipient mothers, and their infants. However, a review of the literature found that the lack of HCP's involvement is due to lack of awareness on the use of non-biological mother's milk (Perrin et al., 2016; Reyes-Foster et al., 2015) and bottle feeding seems to be an ideal option for infant feeding difficulties (McCloskey \& Karandikar, 2018; Perrin et al., 2016; Reyes-Foster et al., 2015). Nevertheless, a few studies in the literature reported that mothers received support from health care providers in terms of information, referrals, and emotional support (Gribble, 2014c; McCloskey \& Karandikar, 2018; Papanicolaou, 2013). Lactation 
consultants, midwives, childbirth instructors, doulas, and nurses were among the sources cited in the studies.

\section{Problems with accessing human milk from the milk} bank

Difficulties in accessing human milk from the milk bank stem from the unavailability of the milk bank and inaccessibility of breastmilk from the milk bank. The unavailability of the milk bank is the main factor that influences a mother's decision to engage in milk exchange via offline or online methods (Gribble, 2013). Additionally, difficulty to access breastmilk due to the policy requirements imposed by the milk bank also causes mothers to choose milk sharing over milk banking (McCloskey \& Karandikar, 2018). Another reason identified is the women's desire to preserve the quality of breastmilk, thus preferring to share milk with a peer rather than milk banking, as they believe the pasteurization process destroys its nutritive contents (Gribble, 2013; McCloskey \& Karandikar, 2018; Perrin et al., 2016).

\section{Conflicting issues from a religious perspective}

Different opinions on milk banking and milk sharing as expressed via social networks were reported in Onat and Karakoç's (2019) study. More than half of the milk donors and recipients in their study preferred the milk bank to milk sharing due to concerns regarding the legal relationship. However, a few citations reported on how participants negotiated their religious understanding while practicing milk sharing (Gribble, 2014c, 2018; Thorley, 2012; Onat \& Karakoç, 2019).

\section{Discussion}

Research into breastmilk sharing via social networks started to become more intense in 2012, which corresponds with wide internet coverage, extensive use of internet-capable smartphones and mobile social apps (Noyes, 2020). As a result, the growing number of social network users have greatly influenced milk exchange. This review was based on 25 studies in three countries / regions: the United States and North America, Australia, and Turkey. The fact that research into milk sharing activity was mainly conducted in Western countries could be explained by the early development of information technology and wider access to the internet in those countries (Baker, 2013).

However, there is inconsistency in the concept of milk sharing activity as it interchangeably refers to the blended concept of breastmilk donation and wet nursing practice to overcome the ethical issues surrounding milk banking specifically in the Muslim community (Al-Naqeeb et al., 2000; Hsu et al., 2012). Wet nursing is the practice of a non-biological mother directly breastfeeding another woman's child while receiving remuneration for her lactation labour (AlHreashy, 2018). On the other hand, milk donation refers to the collection and distribution of expressed breast milk in the institutionalized milk bank. Therefore, it must be done under medical prescription where an eligible donor needs to be screened and the milk donated must undergo the pasteurization process before distribution to the eligible infant (DeMarchis et al., 2017).

Milk sharing was identified as being predominantly practiced by young mothers within the reproductive age group, which is consistent with global statistics reporting that $76 \%$ of social network users are women aged 25 to 34 years (Noyes, 2020). This age distribution falls within the active reproductive age group (Oats \& Abrahams, 2015). People in this age group, known as Generation Y or digital natives, are open-minded and expressive; they depend on social networking as the preferred and powerful communication tool (Woodman \& Wyn, 2014). Moreover, it is undeniable that information technology and social networks play a significant role in promoting and educating the public on breastfeeding. This is supported by mothers' enthusiasm to seek information which is augmented by internet literacy (Sulaiman et al., 2016).

The findings of this review suggest that the public are aware of the importance of breastmilk for infant survival, and the idea of breastmilk donation is becoming more acceptable. The findings provide a new perspective on the use of non-biological mothers' milk. It acknowledges the influencing factors and barriers related to non-maternal nursing, allowing HCPs to understand it from the perspectives of individuals. Besides, the findings are useful in the development of a breastmilk donation framework by reflecting on individual experiences. In terms of clinical practice, this review assists HCPs in educating and supporting mothers through informed decision-making. However, milk sharing activity is still extensively debated in society, specifically among HCPs (Akre et al., 2011). Open discussion on the pros and cons of infant feeding as well as on the protection measures if milk sharing was chosen would be useful. This is consistent with the finding by White et al. (2016) that HCPs and the community need to work together to mitigate the risks as this initiative has a significant impact in normalizing breastfeeding practice in the millennial era.

This review provides evidence for HCPs, especially concerning current issues surrounding infant and child feeding. It seems to be a wakeup call for HCPs to engage with the community using social media which has become the preferred communication platform 
nowadays (Tankovska, 2021). Moreover, milk sharing is a new challenge to the HCPs who are bound by medical ethics, including principles of respect for autonomous decision, non-maleficence, beneficence, and justice (Gribble, 2012). The main concerns are regarding the safety of milk sharing, as well as health and morality issues for the parties involved, that is the donor mother, recipient mother, and their infants. Nonetheless, a series of questions need to be answered by the milk donor and recipient mother such as: Do we need it? Can we rely on the process? What are the implications? What mechanisms can be used to minimize the possible risks? What are the moral issues surrounding this practice?

\section{Knowledge gap}

This review provides an insight into many issues that are worthy of further study. Individual experience in milk sharing is significant since addressing this aspect could assist HCPs in developing a framework for breastmilk donation programs using a multidisciplinary approach that suits the needs of the local context. However, the existing literature is limited to understanding the issue from a Western perspective as research on milk sharing has been conducted mainly in that setting. This justifies the need for understanding individual experiences in other regions. For example, mothers living in Muslim and multiracial countries would perceive and experience milk sharing differently from Western mothers. In addition, reviews on the literature show that too little attention has been paid to the emotional aspects of milk sharing, as well as to religious concerns surrounding this activity. These two aspects are crucial as breastfeeding and motherhood are deeply rooted within religious beliefs and socio-cultural viewpoints. An exploration into conflicting issues of this practice, especially from the religious and health care perspectives, is highly required as the lack of in-depth understanding of these issues would bring negative consequences.

\section{Limitation of study}

This review used the standard framework of conducting a scoping review that allows rigorous and systematic analysis when the subject area had not been reviewed comprehensively. Even though this review included a relatively small number of citations, the search was conducted in three main databases of scientific literature that cover a wide variety of subject areas. Nevertheless, the strengths of this paper lie in several aspects: 1) adherence to standardized scoping review guidelines; 2) consultation with a reference librarian to make maximum use of the search strategy; 3 ) setting the parameters by defining the concept of milk sharing; 4) a systematic and iterative process of data analysis; and 5) team analysis to promote data analysis transparency.

\section{Conclusion}

The use of non-biological mothers' breastmilk is connected to maintaining a child's life, an individual's freedom of belief, and moral values. This topic is extensively debated within society due to its unforeseen implications. Without doubt, milk sharing seems to be the easiest and fastest system as compared to wet nursing and milk banking as the process becomes more accessible and faster with the help of information and technology. This scoping review has identified and summarized the nature of research on milk sharing. This review recognizes the knowledge gap in the research on infant feeding practice. It also provides insights for HCPs on the root causes before implementing an effective action plan by acknowledging individual experience, specifically in breastfeeding promotion and education programs.

\section{Ethical aspects and conflict of interest}

The authors declare that they have no conflict of interest.

\section{Funding}

This research received no specific grant from any funding agency, commercial or not-for-profit sectors.

\section{Acknowledgement}

We would like to thank the librarians of University Putra Malaysia and International Islamic University Malaysia for their assistance in using online databases and executing the search strategies.

\section{Author contributions}

All authors contributed to the study conception and design. Material preparation, data collection and analysis were performed by JN, LK, CT and MS. The draft of the manuscript was written by $\mathrm{JN}$ and commented by LK, CT and MS. The manuscript was critically revised by LK and MS. The final approval was made by LK, CT and MS.

\section{References}

Akre, J. E., Gribble, K. D., \& Minchin, M. (2011). Milk sharing from private practice to public pursuit. International Breastfeeding Journal, 6, 8. https://doi.org/10.1186/17464358-6-8

AlHreashy, F. (2018). Non-maternal nursing in the Muslim community: a health perspective review. Journal of Clinical Neonatology, 7(4), 191-197. 
https://doi.org/10.4103/jen.jcn_55_18

Al-Naqeeb, N. A., Azab, A., Eliwa, M. S., \& Mohammed, B. Y. (2000). The introduction of breast milk donation in a Muslim country. Journal of Human Lactation, 16(4), 346350. https://doi.org/10.1177/089033440001600412.

Baker, S. (2013). Conceptualising the use of Facebook in ethnographic research: as tool, as data and as context. Ethnography and Education, 8(2), 131-145. https://doi.org/10.1080/17457823.2013.792504.

Bawany, M., Milhan, A., \& Padela, A. (2016). The unbreakable relationship. Are Muslims aware of the Islamic bioethical perspectives on human breast milk banking? Islamic Horizons, 48-51.

Campbell, L. (1989). Wet-nurses in early modern England: some evidence from the Townshend archive. Medical History, 33(3), 360-370. https://doi.org/10.1017/s0025727300049607

Carter, S. K., \& Reyes-Foster, B. M. (2016). Pure gold for broken bodies: discursive techniques constructing milk banking and peer milk sharing in U.S. news. Symbolic Interaction, 39(3), 353-373. https://doi.org/10.1002/symb.233

Carter, S. K., Reyes-Foster, B. M., \& Carter, J. S. (2018). "Breast is best, donor next": peer breastmilk sharing in contemporary western motherhood. Sociological Inquiry, 88(4), 673-695. https://doi.org/10.1111/soin.12227

Carter, S. K., Reyes-Foster, B., \& Rogers, T. L. (2015). Liquid gold or Russian roulette? Risk and human milk sharing in the US news media. Health, Risk and Society, 17(1), 30-45. https://doi.org/10.1080/13698575.2014.1000269

Cassar-Uhl, D., \& Liberatos, P. (2018). Use of shared milk among breastfeeding mothers with lactation insufficiency. Maternal and Child Nutrition, 14(Suppl 6), e12594. https://doi.org/10.1111/mcn.12594

DeMarchis, A., Israel-Ballard, K., Mansen, K. A., \& Engmann, C. (2017). Establishing an integrated human milk banking approach to strengthen newborn care. Journal of Perinatology, 37(5), 469-474.

https://doi.org/10.1038/jp.2016.198

Eats on Feets - Community Breastmilk Sharing. (2010). Eats on Feets. Retrieved August 13, 2020, from

https://www.eatsonfeets.org/.

Fulminante, F. (2015). Infant feeding practices in Europe and the mediterranean from prehistory to the middle ages: a comparison between the historical sources and bioarchaeology. Childhood in the Past, 8(1), 24-47. https://doi.org/10.1179/1758571615z.00000000026

Gribble, K. D. (2012). Biomedical ethics and peer-to-peer milk sharing. Clinical Lactation, 3(3), 108-111. https://doi.org/10.1891/215805312807022941.

Gribble, K. D. (2013). Peer-to-peer milk donors' and recipients' experiences and perceptions of donor milk banks. Journal of Obstetric, Gynecologic, and Neonatal Nursing, 42(4), 451-461. https://doi.org/10.1111/1552-6909.12220

Gribble, K. D. (2014a). "I'm happy to be able to help": Why women donate milk to a peer via internet-based milk sharing networks. Breastfeeding Medicine, 9(5), 251-256.

https://doi.org/10.1089/bfm.2014.0009

Gribble, K. D. (2014b). “A better alternative": Why women use peer-to-peer shared milk. Breastfeeding Review, 22(1), 1121.

Gribble, K. D. (2014c). Perception and management of risk in internet-based peer-to-peer milk-sharing. Early Child Development and Care, 184(1), 84-98. https://doi.org/10.1080/03004430.2013.772994

Gribble, K. D. (2018). "Someone's generosity has formed a bond between us": Interpersonal relationships in internetfacilitated peer-to-peer milk sharing. Maternal and Child Nutrition, 14(Suppl 6), e12575.

https://doi.org/10.1111/mcn.12575

Hawker, S., Payne, S., Kerr, C., Hardey, M., \& Powell, J. (2002). Appraising the evidence: reviewing disparate data systematically. Qualitative Health Research, 12(9), 12841299. https://doi.org/10.1177/1049732302238251

Hsu, H. T., Fong, T. V., Hassan, N. M., Wong, H. L., Rai, J. K., \& Khalid, Z. (2012). Human milk donation is an alternative to human milk bank. Breastfeeding Medicine, 7(2), 118-122. https://doi.org/10.1089/bfm.2011.0006

Keim, S. A., McNamara, K. A., Jayadeva, C. M., Braun, A. C., Dillon, C. E., \& Geraghty, S. R. (2014). Breast milk sharing via the internet: the practice and health and safety considerations. Maternal and Child Health Journal, 18(6), 1471-1479. https://doi.org/10.1007/s10995-013-1387-6

MacDonald, T., Noel-Weiss, J., West, D., Walks, M., Biener, M., Kibbe, A., \& Myler, E. (2016). Transmasculine individuals' experiences with lactation, chestfeeding, and gender identity: a qualitative study. BMC Pregnancy and Childbirth, 16, 106. https://doi.org/10.1186/s12884-0160907-y.

McCloskey, R. J., \& Karandikar, S. (2018). A liberation health approach to examining challenges and facilitators of peer-topeer human milk sharing. Journal of Human Lactation, 34(3), 438-447. https://doi.org/10.1177/0890334418771301

Noyes, D. (2020, October 29). The top 20 valuable Facebook statistics - updated October 2020. Zephoria Inc. https://zephoria.com/top-15-valuable-facebook-statistics/

Oats, J., \& Abraham, S. (2015). Llewellyn-Jones Fundamentals of Obstetrics and Gynaecology (10th ed). Elsevier.

Onat, G., \& Karakoç, H. (2019). Informal breast milk sharing in a Muslim country: the frequency, practice, risk perception, and risk reduction strategies used by mothers. Breastfeeding Medicine, 14(8), 597-602.

https://doi.org/10.1089/bfm.2019.0027

O’Sullivan, E. J., Geraghty, S. R., \& Rasmussen, K. M. (2016). Informal human milk sharing: A Qualitative Exploration of the Attitudes and Experiences of Mothers. Journal of Human Lactation, 32(3), 416-424.

https://doi.org/10.1177/0890334416651067

Palmquist, A. E. L., \& Doehler, K. (2014). Contextualizing online human milk sharing: structural factors and lactation disparity among middle income women in the U.S. Social Science and Medicine, 122, 140-147. https://doi.org/10.1016/j.socscimed.2014.10.036.

Palmquist, A. E. L., \& Doehler, K. (2016). Human milk sharing practices in the U.S. Maternal and Child Nutrition, 12(2), 278-290. https://doi.org/10.1111/mcn.12221.

Papanicolaou, A. (2013). The online sharing of human milk: A content analysis [Unpublished Doctoral thesis, Queen's University], QSpace. http://hdl.handle.net/1974/8132

PATH. (2019). Strengthening human milk banking: A resource toolkit for establishing \& integrating human milk bank programs. A Global Implementation Framework. PATH. https://www.path.org/programs/maternal-newborn-childhealth-and-nutrition/strengthening-human-milk-bankingresource-toolkit-0/

Perrin, M. T., Goodell, L. S., Allen, J. C., \& Fogleman, A. (2014). A mixed-methods observational study of human milk 
sharing communities on Facebook. Breastfeeding Medicine, 9(3), 128-134. https://doi.org/10.1089/bfm.2013.0114

Perrin, M. T., Goodell, L. S., Fogleman, A., Pettus, H., Bodenheimer, A. L., \& Palmquist, A. E. L. (2016). Expanding the supply of pasteurized donor milk: understanding why peer-to-peer milk sharers in the United States do not donate to milk banks. Journal of Human Lactation, 32(2), 229-237. https://doi.org/10.1177/0890334415627024

Peters, M. D. J., Godfrey, C. M., McInerney, P., Soares, C. B., Khalil, H., \& Parker, D. (2015). The Joanna Briggs Institute reviewers' manual 2015. Methodology for JBI scoping reviews. The Joanna Briggs Institute.

Rahbari, L. (2020). Milk kinship and the maternal body in Shi'a Islam. Open Theology, 6(1), 43-53. https://doi.org/10.1515/opth-2020-0006

Reyes-Foster, B. M., \& Carter, S. K. (2018). "That's not the milk sharing I'm doing": responses to a pediatrics article from women who milk share. Human Organization, 77(3), 262-272. https://doi.org/10.17730/0018-7259.77.3.262

Reyes-Foster, B. M., Carter, S. K., \& Hinojosa, M. S. (2015). Milk sharing in practice: a descriptive analysis of peer breastmilk sharing. Breastfeeding Medicine, 10(5), 263-269. https://doi.org/10.1089/bfm.2015.0009

Reyes-Foster, B. M., Carter, S. K., \& Hinojosa, M. S. (2017). Human milk handling and storage practices among peer milk-sharing mothers. Journal of Human Lactation, 33(1), 173-180. https://doi.org/10.1177/0890334416678830

Schafer, E. J., Ashida, S., \& Palmquist, A. E. L. (2018). Psychosocial dimensions of human milk sharing. Maternal and Child Nutrition, 14(Suppl 6), e12606. https://doi.org/10.1111/mcn.12606

Smith, A. L. (2017). Becoming-(breastfeeding-) woman: women's body practices and experiences at the intersection of breastfeeding, milk insufficiency, and milk sharing [Unpublished doctoral thesis, The University of Queensland]. UQ eSpace.

https://doi.org/10.14264/uql.2017.732

Stevens, E. E., Patrick, T. E., \& Pickler, R. (2009). A history of infant feeding. The Journal of Perinatal Education, 18(2), 32-39. https://doi.org/10.1624/105812409X426314.

Sulaiman, Z., Liamputtong, P., \& Amir, L. H. (2016). The enablers and barriers to continue breast milk feeing in women returning to work. Journal of Advanced Nursing , 72(4), 825835. https://doi.org/10.1111/jan.12884

Tanskovska, H. (2021, February 9). Global social networks ranked by number of users 2021. Statista. Retrieved March 5, 2021 from

https://www.statista.com/statistics/272014/global-socialnetworks-ranked-by-number-of-users/

Thorley, V. (2012). Mothers' experiences of sharing breastfeeding or breastmilk, part 2: the early 21 st century. Nursing Reports, 2(1), e2. https://doi.org/10.4081/nursrep.2012.e2

White, B., White, J., Giglia, R., \& Tawia, S. (2016). Feed Safe: a multidisciplinary partnership approach results in a successful mobile application for breastfeeding mothers. Health Promotion Journal of Australia, 27(2), 111-117. https://doi.org/10.1071/he15114.

Woodman, D., \& Wyn, J. (2014). Youth and generation: Rethinking change and inequality in the lives of young people ( $1^{\text {st }}$ ed.). SAGE. 